\title{
Local Modelling of Nonlinear Dynamic Systems Using Direct Weight Optimization
}

\author{
Jacob Roll, Alexander Nazin, Lennart Ljung \\ Division of Automatic Control \\ Department of Electrical Engineering \\ Linköpings universitet, SE-581 83 Linköping, Sweden \\ WWW: http://www. control.isy.liu.se \\ E-mail: roll,ljung@isy.liu.se, nazine@ipu.rssi.ru
}

January 9, 2003

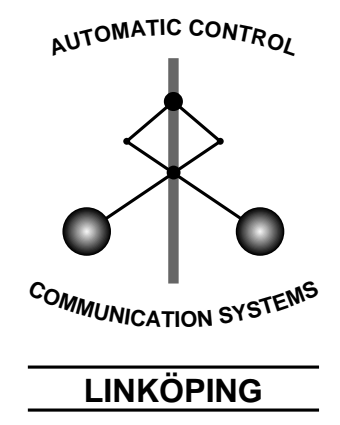

Report no.: LiTH-ISY-R-2483

Submitted to SYSID'03

Technical reports from the Control \& Communication group in Linköping are available at http://www. control.isy.liu.se/publications. 



\title{
Local Modelling of Nonlinear Dynamic Systems Using Direct Weight Optimization
}

\author{
Jacob Roll;, Alexander Nazin†, Lennart Ljung*
}

January 9, 2003

\begin{abstract}
Local models and methods construct function estimates or predictions from observations in a local neighborhood of the point of interest. The bandwidth, i.e., how large the local neighborhood should be, is often determined based on asymptotic analysis. In this paper, an alternative, non-asymptotic approach that minimizes a uniform upper bound on the mean square error for a linear estimate is used. It is shown that the estimator is obtained from a quadratic program, that an automatic bandwidth selection is obtained, and that the approach can be seen as a local version of fitting affine models to data. Finally, the approach is applied to two benchmark systems.
\end{abstract}

\section{Introduction: Local Models}

Non-linear black box models of dynamical systems have long been of central interest in system identification, see, e.g., the survey [10]. In the control community mostly models of function expansion type have been applied, like Artificial Neural Network (ANN) models, wavelets, and (neuro-)fuzzy models. In statistics, the interest has been focused on various local methods, like kernel methods, [6], local polynomial approaches, [3] and trees, [1].

A local model or method builds the function estimate or prediction from observations in a local neighborhood of the point of interest. Also most function expansion methods are of this character: A radial basis neural network is built up from basis functions with local support, and the standard sigmoidal (one hidden layer feed-forward) network is local around certain hyperplanes in the regressor space.

No matter what type of local modelling approach is taken, the central problem is the bandwidth question:

- How big should the local neighborhood be?

This is a variant of the classical bias/variance trade-off. Intuitively, it is clear that the answer must depend on three items:

\footnotetext{
*Div. of Automatic Control, Linköping University, SE-58183 Linköping, Sweden, e-mail: roll, ljung@isy.liu.se

${ }^{\dagger}$ Institute of Control Sciences, Profsoyuznaya str., 65, 117997 Moscow, Russia, e-mail: nazine@ipu.rssi.ru
} 
1. How many data are available (and how are they distributed)?

2. How smooth is the function surface (supposed to be)?

3. How much noise is there in the observations?

This problem has been studied extensively in the statistical literature, and there are several solutions based on asymptotic (in the number of observations) analysis $[3,2]$.

In this paper, another solution that is not based on the asymptotic behavior of the estimates is proposed. Based on a smoothness measure and noise variance, a uniform upper bound is computed of the mean square error (MSE) of a linear estimate, as a function of the estimator parameters. This upper bound is then minimized. It turns out that this problem can be reformulated as a quadratic programming (QP) problem, which can be solved efficiently. It also turns out that this solution has many of the key features of the asymptotically optimal estimators, but for finite number of observations it produces better guaranteed error bounds. The method will be called a direct weight optimization (DWO) approach, since the weights of the linear estimator are directly optimized, without any reference to kernels or parametric models.

The basic idea was presented earlier in [8] for approximation of scalar functions, where examples also showed that it is sometimes superior to an asymptotically based local linear estimator. In this paper, the approach is extended to multivariate functions and put in a system identification context.

The paper is organized as follows: In Section 2, the problem is stated, and a very brief overview of some of the existing local approaches is given. The new approach is described in Section 3, and some of its most important properties are derived in Section 4. In Section 5 some examples are given of how the DWO approach can be used for prediction. Some extensions are described in Section 6 and conclusions are given in Section 7 .

\section{Problem Statement}

Consider the problem of estimating the value $f\left(\varphi_{0}\right)$ of an unknown multivariate function $f: \mathbf{R}^{n} \rightarrow \mathbf{R}$ at a given point $\varphi_{0}$, given a set of input-output pairs $\left\{\left(\varphi_{k}, y_{k}\right)\right\}_{k=1}^{N}$, coming from the relation

$$
y_{k}=f\left(\varphi_{k}\right)+e_{k}
$$

Assume that the function $f$ is continuously differentiable, and that there is a Lipschitz constant $L$ such that

$$
\left\|\nabla f\left(\varphi_{1}\right)-\nabla f\left(\varphi_{2}\right)\right\| \leq L\left\|\varphi_{1}-\varphi_{2}\right\|
$$

where $\|\cdot\|$ is the Euclidean norm. Denote the class of functions satisfying these assumptions by $\mathcal{F}_{L}$.

The noise terms $e_{k}$ are independent random variables with $E e_{k}=0$ and $E e_{k}^{2}=\sigma_{k}^{2}$. Both $L$ and $\sigma_{k}$ are assumed to be positive constants, given a priori. The notation

$$
\tilde{\varphi}_{k}=\varphi_{k}-\varphi_{0}
$$

and $X=\left(\varphi_{1}, \ldots, \varphi_{N}\right)$ will also be used. 
The given problem arises, e.g., when predicting outputs from a system of NARX structure [10], where the system dynamics is known to satisfy (2). In this case, $\varphi_{k}$ is composed of previous inputs $u_{k-\tau}$ and outputs $y_{k-\tau}$, i.e.,

$$
\varphi_{k}=\left(\begin{array}{llllll}
y_{k-1} & \ldots & y_{k-n_{a}} & u_{k-1} & \ldots & u_{k-n_{b}}
\end{array}\right)^{T}
$$

This fits in especially well within the "Model-On-Demand" (MOD) framework (see $[11])$.

A common approach in statistics for estimation problems of this kind is to use a linear estimator in the form

$$
\hat{f}\left(\varphi_{0}\right)=\sum_{k=1}^{N} w_{k} y_{k}
$$

where $\hat{f}\left(\varphi_{0}\right)$ is our estimate of $f\left(\varphi_{0}\right)$. The problem then reduces to finding good weights $w_{k}$, which give reasonably small bias and variance of the estimate over the class $\mathcal{F}_{L}$. A common measure of the performance of the estimator is the mean squared error (MSE) [3] defined by

$$
\operatorname{MSE}\left(\hat{f}, \varphi_{0}\right)=E\left[\left(\hat{f}\left(\varphi_{0}\right)-f\left(\varphi_{0}\right)\right)^{2} \mid X\right]
$$

Often one tries to minimize the worst-case MSE, i.e., achieve the linear minimax risk, defined by

$$
R(X, L)=\inf _{\hat{f}\left(\varphi_{0}\right)} \sup _{f \in \mathcal{F}_{L}} E\left[\left(\hat{f}\left(\varphi_{0}\right)-f\left(\varphi_{0}\right)\right)^{2} \mid X\right]
$$

where the infimum is taken over all linear estimators.

A classic family of methods are the kernel methods, where a kernel function $K$ is used to determine the weights. An example is the Nadaraya-Watson estimator $[6,12]$. Common choices of kernels are the Gaussian kernel and the spherical Epanechnikov kernel

$$
K(u)=C\left(1-\|u\|^{2}\right)_{+}
$$

where $(\cdot)_{+}=\max \{\cdot, 0\}$ and $C$ is a normalization constant. The width of the kernel is specified by introducing a bandwidth matrix $H$, and using the kernel $K_{H}(u)=\operatorname{det}(H)^{-1} K\left(H^{-1} u\right)$ when calculating the weights. In the setting of this paper (i.e., where all that is known is that $f$ satisfies $(2)$ ), it is sufficient to consider $H=h I$.

Another popular approach is the local polynomial modelling approach [3], where the estimator is determined by locally fitting a polynomial to the given data. A weighted least-squares problem is obtained, which for a first-order polynomial takes the form

$$
\hat{\beta}=\arg \min _{\beta} \sum_{k=1}^{N} K_{H}\left(\tilde{\varphi}_{k}\right)\left(y_{k}-\left(\beta_{0}+\beta_{1}^{T} \tilde{\varphi}_{k}\right)\right)^{2}
$$

Here, $\hat{f}\left(\varphi_{0}\right)=\hat{\beta}_{0}$, and the weights $w_{k}$ in (5) are thus implicitly determined. The bandwidth $h$ can be determined to asymptotically achieve the linear minimax risk (see [3] for details). 
The different methods given in this section for choosing the weights $w_{k}$ in the linear estimator (5) were all justified using asymptotic arguments, as $N \rightarrow \infty$. However, in reality only a finite number of data is given. Furthermore, these data may be sparsely and non-uniformly distributed. This might deteriorate the performance of the estimation methods.

In the following section, a non-asymptotic approach for determining the weights will be presented, based on a uniform (over $\mathcal{F}_{L}$ ) upper bound on the MSE (and hence on the linear minimax risk).

\section{A Direct Weight Optimization Approach}

As in the previous section, a linear estimator in the form (5) will be considered. For the bias to be bounded over $\mathcal{F}_{L}$, the following requirements on the weights $w_{k}$ are needed (see [9]):

$$
\begin{array}{r}
\sum_{k=1}^{N} w_{k}=1 \\
\sum_{k=1}^{N} w_{k} \tilde{\varphi}_{k}=0
\end{array}
$$

Under these restrictions, any linear function is estimated with zero bias. Note also that the local linear estimator described in the last section will automatically satisfy these requirements.

From (2), one can obtain

$$
f(\varphi)=f\left(\varphi_{0}\right)+\nabla^{T} f\left(\varphi_{0}\right) \tilde{\varphi}+\frac{c(\varphi)}{2}\|\tilde{\varphi}\|^{2}
$$

for some $c(\varphi),|c(\varphi)| \leq L$. Using all these equations, one gets

$$
\hat{f}\left(\varphi_{0}\right)=f\left(\varphi_{0}\right)+\sum_{k=1}^{N} w_{k} \frac{c\left(\varphi_{k}\right)}{2}\left\|\tilde{\varphi}_{k}\right\|^{2}+\sum_{k=1}^{N} w_{k} e_{k}
$$

and an upper bound on the MSE (over $\mathcal{F}_{L}$ )

$$
\begin{aligned}
E\left[\left(\hat{f}\left(\varphi_{0}\right)\right.\right. & \left.\left.-f\left(\varphi_{0}\right)\right)^{2} \mid X\right] \\
& \leq \frac{L^{2}}{4}\left(\sum_{k=1}^{N}\left\|\tilde{\varphi}_{k}\right\|^{2}\left|w_{k}\right|\right)^{2}+\sum_{k=1}^{N} w_{k}^{2} \sigma_{k}^{2}
\end{aligned}
$$

Note that this bound is tight and attained by a paraboloid with the Hessian $\nabla^{2} f(\varphi)=L \cdot I$ if the weights $w_{k}$ are non-negative.

It is now natural to minimize the upper bound in (12). Hence, the values of 
$w_{k}$ are chosen to minimize the following convex optimization problem:

$$
\begin{aligned}
\min _{w_{k}} & \frac{L^{2}}{4}\left(\sum_{k=1}^{N}\left\|\tilde{\varphi}_{k}\right\|^{2}\left|w_{k}\right|\right)^{2}+\sum_{k=1}^{N} w_{k}^{2} \sigma_{k}^{2} \\
\text { subj. to } & \sum_{k=1}^{N} w_{k}=1 \\
& \sum_{k=1}^{N} w_{k} \tilde{\varphi}_{k}=0
\end{aligned}
$$

This problem is equivalent to the following QP:

$$
\begin{aligned}
\min _{w_{k}, s_{k}} & \frac{L^{2}}{4}\left(\sum_{k=1}^{N}\left\|\tilde{\varphi}_{k}\right\|^{2} s_{k}\right)^{2}+\sum_{k=1}^{N} s_{k}^{2} \sigma_{k}^{2} \\
\text { subj. to } & s_{k} \geq w_{k} \\
& s_{k} \geq-w_{k} \\
& \sum_{k=1}^{N} w_{k}=1 \\
& \sum_{k=1}^{N} w_{k} \tilde{\varphi}_{k}=0
\end{aligned}
$$

The equivalence between (13) and (14) can be shown as follows: Given a feasible solution $w=\left(w_{1}, \ldots, w_{N}\right)$ to $(13)$, a feasible solution to (14) with the same value of the objective function can easily be obtained by using the same $w$ and $s_{k}=$ $\left|w_{k}\right|$. Hence (14) is a relaxation of (13), and it suffices to show that when the optimal value of (14) is reached, then $s_{k}=\left|w_{k}\right|$ for all $k=1, \ldots, N$. Suppose, e.g., that $s_{1}>\left|w_{1}\right|$. Then, without changing any other variables, the value of the objective function can be reduced by decreasing $s_{1}$. This can be seen by observing that the coefficient before $s_{1}$ in the first sum of the objective function is non-negative, and the coefficient before $s_{1}^{2}$ in the second sum is positive, so decreasing $s_{1}$ will decrease the objective function. Therefore, when the objective function will reach its minimum, then $s_{k}=\left|w_{k}\right|$, and the equivalence is shown.

It should be pointed out, that the fact that the upper bound in (12) is tight for non-negative weights $w_{k}$ does not necessarily mean that minimizing (14) yields the weights that minimize the worst-case MSE, even if the resulting weights are positive. The reason for this is that a subset of the weights that really minimize the worst-case MSE may be negative, and so the upper bound (12) is not tight for these weights. However, preliminary experimental results show that the solution of (14) gives an upper bound which is mostly within a few percents from the optimal worst-case MSE.

Solving the QP (14) can be done very efficiently using standard solvers, e.g., CPLEX [4].

\section{Some Basic Properties of the DWO Approach}

Since the DWO approach minimizes an upper bound on the linear minimax risk, one would expect that the weights $w_{k}$ would asymptotically converge to 
the weights of the local linear estimator using the Epanechnikov kernel with an asymptotically optimal bandwidth [3]. This is also the case under certain assumptions, as the following theorem shows. For a proof of the result, see [9].

Theorem 4.1 Consider the problem of estimating an unknown scalar function $f: \mathbf{R} \rightarrow \mathbf{R}, f \in \mathcal{F}_{L}$ at a given internal point $\varphi_{0} \in(-1 / 2,1 / 2)$ under a fixed, equidistant design model as follows

$$
\varphi_{k}=\frac{k-1}{N-1}-\frac{1}{2}, \quad k=1, \ldots, N
$$

and with $\sigma_{k}=\sigma>0$ for all $k=1, \ldots, N$. Let

$$
w^{o p t}=\left(w_{1}^{o p t}, \ldots, w_{N}^{o p t}\right)
$$

be the minimum point of the upper bound function

$$
U(w)=\left(\frac{L}{2} \sum_{k=1}^{N}\left|w_{k}\right|\left|\tilde{\varphi}_{k}\right|^{2}\right)^{2}+\sigma^{2} \sum_{k=1}^{N} w_{k}^{2}
$$

subject to the constraints (10). Then asymptotically, as $N \rightarrow \infty$,

$$
w_{k}^{o p t} \approx \frac{3}{4} C_{N}\left(1-\left(\frac{\tilde{\varphi}_{k}}{h_{N}}\right)^{2}\right)_{+}, \quad k=1, \ldots, N
$$

where

$$
C_{N} \asymp \frac{1}{N h_{N}}, \quad h_{N} \asymp\left(\frac{15 \sigma^{2}}{L^{2} N}\right)^{1 / 5} \quad \text { as } N \rightarrow \infty
$$

Hence, the optimal weights (17) approximately coincide with related asymptotically optimal weights and bandwidth of the local polynomial estimator for the worst case function in $\mathcal{F}_{L}$.

Here $a_{N} \asymp b_{N}$ means asymptotic equivalence of two real sequences $\left(a_{N}\right)$ and $\left(b_{N}\right)$, that is $a_{N} / b_{N} \rightarrow 1$ as $N \rightarrow \infty$.

Remark. When the data are symmetrically distributed around $\varphi_{0}$, e.g., when $\varphi_{0}=0$, the relation (17) will hold exactly also for finite $N$. In other words, the non-zero weights will lie along a parabola given by (17), with an asymptotic bandwidth given by (18).

Another interesting feature of the DWO approach is that in most cases, the weights $w_{k}$ corresponding to $\varphi_{k}$ lying beyond a certain distance from $\varphi_{0}$ will be zero. This can be regarded as an automatic bandwidth selection, which means that the user does not have to bother about how many of the samples should be included in the estimator. In fact, the following theorem holds:

Theorem 4.2 Suppose that the problem (14) is feasible, and $\sigma_{k}=\sigma>0$ for all $k=1, \ldots, N$. Then there exist $\mu_{1} \in \mathbf{R}, \mu_{2} \in \mathbf{R}^{n}$, and $\mu_{3} \in \mathbf{R}, \mu_{3} \geq 0$, such that for an optimal solution $\left(s^{o p t}, w^{o p t}\right)$, it holds that

$$
\begin{aligned}
& w_{k}^{o p t}= \\
& \begin{cases}\mu_{1}+\mu_{2}^{T} \tilde{\varphi}_{k}-\mu_{3}\left\|\tilde{\varphi}_{k}\right\|^{2}, & \mu_{3}\left\|\tilde{\varphi}_{k}\right\|^{2} \leq \mu_{1}+\mu_{2}^{T} \tilde{\varphi}_{k} \\
0, & \left|\mu_{1}+\mu_{2}^{T} \tilde{\varphi}_{k}\right| \leq \mu_{3}\left\|\tilde{\varphi}_{k}\right\|^{2} \\
\mu_{1}+\mu_{2}^{T} \tilde{\varphi}_{k}+\mu_{3}\left\|\tilde{\varphi}_{k}\right\|^{2}, & \mu_{1}+\mu_{2}^{T} \tilde{\varphi}_{k} \leq-\mu_{3}\left\|\tilde{\varphi}_{k}\right\|^{2}\end{cases}
\end{aligned}
$$




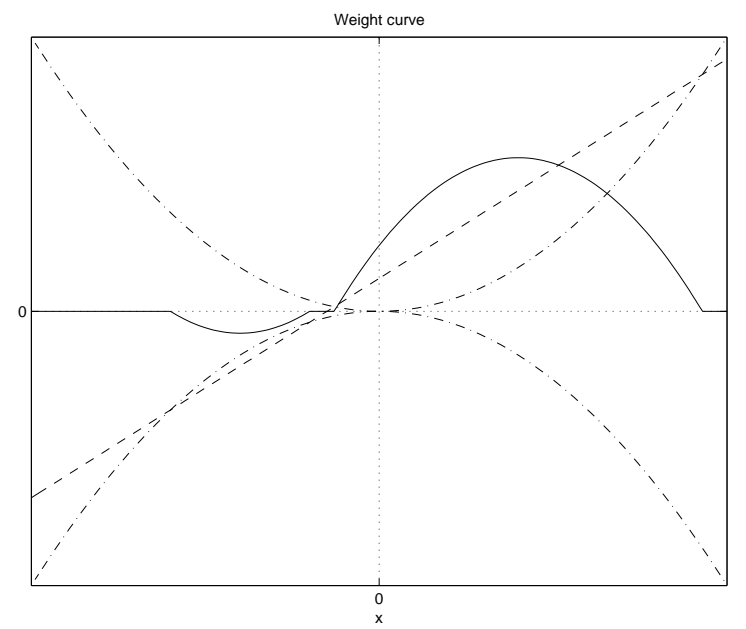

Figure 1: Principal shape of the weight curve (solid curve). The dash-dotted parabolas are $\pm \mu_{3} \tilde{\varphi}^{2}$, and the dashed line is $\mu_{1}+\mu_{2}^{T} \tilde{\varphi}$. (The weight curve is scaled by a factor 4 to make the figure more clear.)

Proof: To appear. See [9] for the scalar case.

Figure 1 shows, for the scalar case, the principal shape of the curve along which the weights $w_{k}^{o p t}$ are placed. When $\mu_{3}\left\|\tilde{\varphi}_{k}\right\|^{2} \leq \mu_{1}+\mu_{2}^{T} \tilde{\varphi}_{k}$ (which in the figure corresponds to the dashed line being above the upper dash-dotted parabola), the weights will be positive. When $\mu_{1}+\mu_{2}^{T} \tilde{\varphi}_{k} \leq-\mu_{3}\left\|\tilde{\varphi}_{k}\right\|^{2}$ (the dashed line is below the lower dash-dotted parabola), the weights are negative, and otherwise they are zero.

The last result opens up for a possible reduction of the computational complexity: Since many of the weights $w_{k}$ will be zero, one can already beforehand exclude data that will most likely correspond to zero weights, thus making the QP (14) considerably smaller. Having solved (14), one can easily check whether or not the excluded weights really should be zero, by checking if the excluded data points satisfy $\left|\mu_{1}+\mu_{2}^{T} \tilde{\varphi}_{k}\right| \leq \mu_{3}\left\|\tilde{\varphi}_{k}\right\|^{2}$ (the middle case of (19)). For more details, see [9].

A third property to note is that, when $L=0$, the optimal weights from (13) equal the weights implicitly obtained from $(9)$, with $K_{H}\left(\tilde{\varphi}_{k}\right)$ replaced by $1 / \sigma_{k}^{2}$, just as one would expect. Since the latter approach gives a global affine model of ARX type (which is independent of $\varphi_{0}$ ), the weights obtained from (13) with $L=0$ can also be seen as corresponding to an underlying global affine model. Hence, the proposed DWO approach can be interpreted as an alternative local version of fitting an "affine ARX model" to the data. The property is given in the following theorem:

Theorem 4.3 For $L=0$, (13) gives the same weights as a local linear model given by (9) with $K_{H}\left(\tilde{\varphi}_{k}\right)=1 / \sigma_{k}^{2}$. 
Proof: With $K_{H}\left(\tilde{\varphi}_{k}\right)=1 / \sigma_{k}^{2}$ and

$$
\begin{aligned}
& \beta=\left(\begin{array}{c}
\beta_{0} \\
\beta_{1}
\end{array}\right), \quad \Phi=\left(\begin{array}{ccc}
1 & \ldots & 1 \\
\varphi_{1} & \ldots & \varphi_{N}
\end{array}\right), \\
& Y=\left(\begin{array}{c}
y_{1} \\
\vdots \\
y_{N}
\end{array}\right), \quad Q=\left(\begin{array}{ccc}
1 / \sigma_{1} & & 0 \\
& \ddots & \\
0 & & 1 / \sigma_{N}
\end{array}\right)
\end{aligned}
$$

$\hat{\beta}_{0}$ obtained from (9) can be written

$$
\hat{\beta}_{0}=\mathbf{e}_{1}^{T}\left(\Phi Q^{2} \Phi^{T}\right)^{-1} \Phi Q^{2} Y
$$

where $\mathbf{e}_{1}$ is the first vector of the standard basis. This corresponds to the weights

$$
w^{L L M}=Q^{2} \Phi^{T}\left(\Phi Q^{2} \Phi^{T}\right)^{-1} \mathbf{e}_{1}
$$

On the other hand, if $L=0$, (13) can be formulated as

$$
\begin{aligned}
\min _{w} & w^{T} Q^{-2} w \\
\text { subj. to } & \Phi w=\mathbf{e}_{1}
\end{aligned}
$$

or, letting $v=Q^{-1} w$,

$$
\begin{aligned}
\min _{v} & v^{T} v \\
\text { subj. to } & \Phi Q v=\mathbf{e}_{1}
\end{aligned}
$$

But this is nothing else than finding the least-norm solution of a system of linear equations, which is easily done using the pseduoinverse, i.e., $v=(\Phi Q)^{\dagger} \mathbf{e}_{1}$. Hence

$$
w=Q v=Q^{2} \Phi^{T}\left(\Phi Q^{2} \Phi^{T}\right)^{-1} \mathbf{e}_{1}
$$

which are exactly the same weights as (21).

\section{$5 \quad$ Examples}

Example 5.1 To give an example of the DWO approach, it was applied to an extended version of the linear so-called Åström system [5]:

$$
\begin{aligned}
& y(t)= \\
& \quad 1.5 y(t-1)-0.7 y(t-2)+u(t-1)+0.5 u(t-2) \\
& \quad+\alpha+L_{0}\left(\cos y(t-1)+0.5 u^{2}(t-1)\right)+e(t)
\end{aligned}
$$

First $\alpha$ and $L_{0}$ were set to zero. To get estimation data, $u(t)$ and $e(t)$ were both selected as random Gaussian sequences of length 500, with unit variance. As validation data, 200 samples of noise-free data were selected, with $u(t)$ generated in the same way as for the estimation data. The simulated output for $L=0.01$ is shown in Figure 2 As can be seen, the simulated output follows the true output well $(84.9 \%$ fit). For $L=0$, the result is the same as fitting an affine model using a least-squares criterion, according to Theorem 4.3, giving $85.9 \%$ fit. As 
comparison, a linear ARX model was also estimated, and performed slightly better compared to the other approaches (91.8\% fit), as expected, since the true system was linear.

Choosing $L_{0}=L=1, \alpha=1$ and using a estimation data sequence of 50000 samples (generated as above), yielded a fit of $52.4 \%$ for the simulated output, as compared to $41.8 \%$ for a linear ARX model (estimated after removing means from the data). The corresponding numbers for one-step-ahead predictions were $94.8 \%$ and $77.5 \%$, respectively.

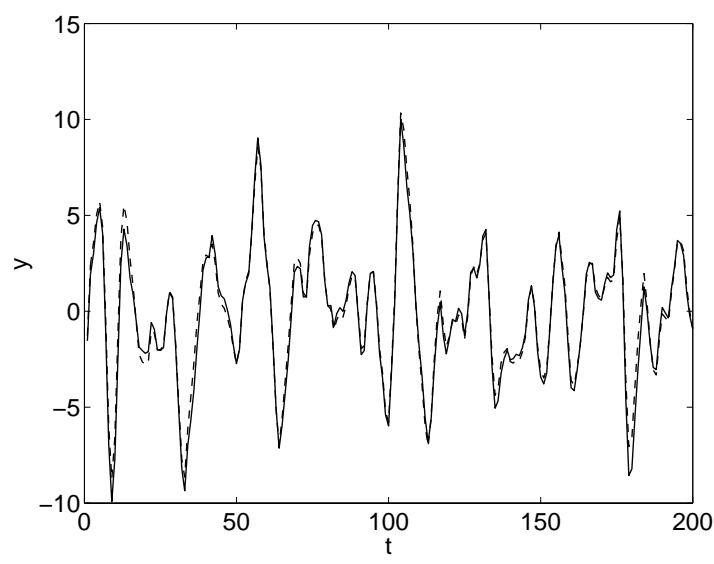

Figure 2: Simulated (solid) and true (dashed) output for system (24) with $L_{0}=0$, modelled using the DWO approach with $L=0.01$.

Example 5.2 As another example, a nonlinear benchmark system proposed by [7] is considered. The system is defined in state-space form by

$$
\begin{aligned}
x_{1}(t+1)= & \left(\frac{x_{1}(t)}{1+x_{1}^{2}(t)}+1\right) \sin x_{2}(t) \\
x_{2}(t+1)= & x_{2}(t) \cos x_{2}(t)+x_{1}(t) e^{-\frac{x_{1}^{2}(t)+x_{2}^{2}(t)}{8}} \\
& +\frac{u^{3}(t)}{1+u^{2}(t)+0.5 \cos \left(x_{1}(t)+x_{2}(t)\right)} \\
y(t)= & \frac{x_{1}(t)}{1+0.5 \sin x_{2}(t)}+\frac{x_{2}(t)}{1+0.5 \sin x_{1}(t)}+e(t)
\end{aligned}
$$

The noise term $e(t)$ is added in accordance with [11] and has a variance of 0.1. The states are assumed not to be measurable, and following the discussion in [11], a NARX331 structure is used to model the system, i.e.,

$$
\begin{aligned}
& \varphi(t)= \\
& (y(t-1) y(t-2) y(t-3) u(t-1) u(t-2) u(t-3))^{T}
\end{aligned}
$$

As estimation data, $N=50000$ samples were generated using a uniformly distributed random input $u(t) \in[-2.5,2.5]$. To validate the model, the input signal

$$
u(t)=\sin \frac{2 \pi t}{10}+\sin \frac{2 \pi t}{25}, \quad t=1, \ldots, 200
$$


was used. Figure 3 shows the simulated output when $L$ was chosen to be 0.1 . The results are reasonable ( $49.7 \%$ fit), although it should be noted that the Lipschitz constant is not known a priori, and is chosen ad hoc to be constant over the entire state-space. In fact, since the real system is not of NARX structure, there might not even exist a such a Lipschitz constant. Therefore, combining the approach with a local estimation of $L$ using an algorithm similar to the bandwidth selection methods in, e.g., [11], would probably improve the results.

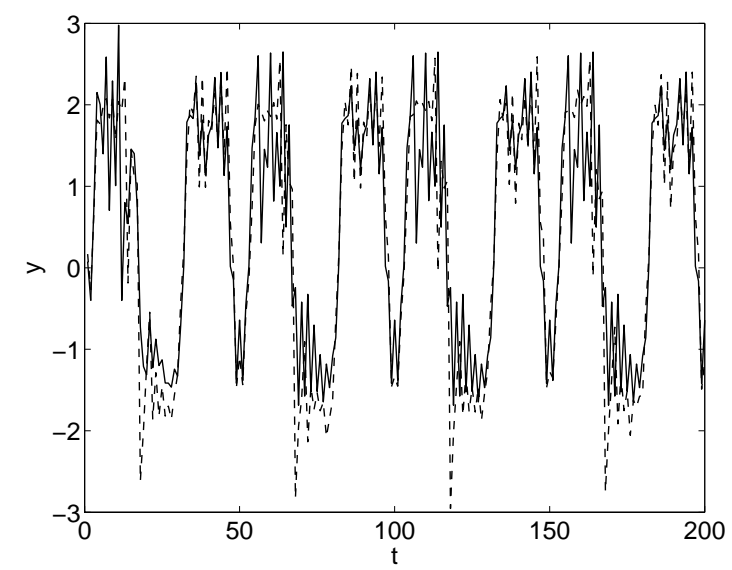

Figure 3: Simulated (solid) and true (dashed) output for system (25), modelled using the DWO approach with $L=0.1$.

\section{$6 \quad$ Extensions}

\subsection{Prior knowledge}

One may think of the constraints (10a) and (10b) as ad hoc, although reasonable. These constraints can be removed if instead a restricted family of functions is considered with some prior knowledge of the function value and its derivative:

$$
\left|f\left(\varphi_{0}\right)-a\right| \leq A, \quad\left|f^{\prime}\left(\varphi_{0}\right)-b\right| \leq B
$$

and formulate the corresponding min-max MSE problem. It can then be shown, see [9], that for sufficiently large $A$ and $B$, the solution will automatically be subject to the constraints (10a) and (10b).

Moreover, if a reasonable linear estimate of the derivative $\nabla f\left(\varphi_{0}\right)$ is formed and plugged in as a known value (with or without error), the resulting estimator will obey (10b). (See [9] for the scalar case.)

\subsection{More smoothness}

The criterion that has been used assumes the function to be once continuously differentiable with a bound on the Lipschitz constant of the derivative. It is quite easy to extend this to any degree of assumed differentiability. The minmax problem will still be reducible to a QP problem, [9]. 


\section{Conclusions}

The proposed non-asymptotic DWO approach to local modelling has a number of interesting features:

- The problem is phrased without any reference to bandwidth. The formulation offers the possibility to use all observations. Theorem 4.2 however shows that there is a bandwidth feature even for a finite number of measurements: Observations outside a certain band carry weights that are exactly zero.

- Although the DWO approach does not give strictly better estimates (in the MSE sense) than, say, the local polynomial approach in all cases, the important point is that the delivered guaranteed MSE bound is better than what other approaches can offer. In practice it is of course only this guaranteed bound that can be used for confidence intervals etc, since the actual MSE depends on the unknown function.

- As is illustrated in [8], the improvement over asymptotically optimal estimates is more pronounced (naturally enough) for fewer data, and more non-uniformly distributed observation points $\varphi_{k}$. For applications to higher regressor dimensions and dynamical systems, this is a very valuable property.

Finally, one may ask how to find the "input values" $L$ and $\sigma_{k}^{2}$ to the algorithms. This is the same problem as for the kernel methods and the local polynomial approach, and has been extensively studied in the statistical literature. In [3] related ideas can be found.

The proposed approach can be used as an alternative to building non-linear black-box models in an MOD fashion and applied to, for example, model predictive control. See [11] for such ideas.

\section{References}

[1] L. Breiman, J. H. Friedman, R. A. Olshen, and C. J. Stone. Classification and Regression Trees. Wadsworth \& Brooks, 1984.

[2] W. S. Cleveland and C. Loader. Smoothing by local regression: Principles and methods. Technical report, AT\&T Bell Laboratories, 600 Mountain Avenue, Murray Hill, NJ 07974, USA, 1994.

[3] J. Fan and I. Gijbels. Local Polynomial Modelling and Its Applications. Chapman \& Hall, 1996.

[4] ILOG, Inc. CPLEX 7.0 User's Manual. Gentilly, France, 2000.

[5] L. Ljung. System Identification: Theory for the User. Prentice-Hall, 2nd edition, 1999.

[6] E. Nadaraya. On estimating regression. Theory of Probability and its Applications, 10:186-190, 1964. 
[7] K. S. Narendra and S.-M. Li. Neural networks in control systems. In P. Smolensky, M. C. Mozer, and D. E. Rumelhart, editors, Mathematical Perspectives on Neural Networks, chapter 11, pages 347-394. Lawrence Erlbaum Associates, 1996.

[8] J. Roll, A. Nazin, and L. Ljung. A non-asymptotic approach to local modelling. In The 41st IEEE Conference on Decision and Control, December 2002 .

[9] J. Roll, A. Nazin, and L. Ljung. Non-asymptotic optimal guaranteed MSE function estimation. Technical Report LiTH-ISY-R-2415, Department of Electrical Engineering, Linköping University, 2002.

[10] J. Sjöberg, Q. Zhang, L. Ljung, A. Benveniste, B. Delyon, P. Y. Glorennec, H. Hjalmarsson, and A. Juditsky. Nonlinear black-box modeling in system identification: a unified overview. Automatica, 31(12):1691-1724, 1995.

[11] A. Stenman. Model on Demand: Algorithms, Analysis and Applications. $\mathrm{PhD}$ thesis, Department of Electrical Engineering, Linköping University, SE-581 83 Linköping, Sweden, 1999.

[12] G. Watson. Smooth regression analysis. Sankhyā, A(26):359-372, 1964. 